\title{
Descrição da população de animais de companhia e epidemiologia da dirofilariose canina em Itacoatiara, município de Niterói, $\mathbf{R J}$
}

\author{
Description of pet population and canine heartworm epidemiology in \\ Itacoatiara, municipality of Niterói, RJ
}

Maria Lúcia Serrão, ${ }^{*}$ Renata Batista, ${ }^{*}$ Elan Paes-de-Almeida, ${ }^{\star *}$ Patrícia Santos, ${ }^{*}$ Yuri Fontenele Melo, ${ }^{\star}$ Norma Labarthe ${ }^{\star \star \star}$

\begin{abstract}
Resumo
Para conhecer a população de cães e gatos de Itacoatiara, Niterói, RJ, realizou-se um censo de janeiro a março de 1995. Determinou-se somente a prevalência da infecção canina por Dirofilaria immitis no bairro, já que em gatos a microfilaremia é rara e transitória e, portanto, sem importância epidemiológica. Demonstrou-se também a ocorrência de casos autóctones da doença. A população de animais de companhia em Itacoatiara correspondeu a $36 \%$ da população humana. Estimou-se que no bairro, em 1995, havia 95 cães infectados por $D$. immitis e portadores de microfilaremia e, portanto, fonte de infecção. A prevalência de casos microfilarêmicos foi $31,7 \%$. Dentre os 91 cães nascidos, criados e que nunca saíram de Itacoatiara (autóctones), 37,8\% eram portadores de microfilárias na circulação.
\end{abstract}

Palavras-chave: Dirofilariose; população de animais de companhia; Dirofilaria immitis.

\begin{abstract}
To evaluate canine and feline populations of Itacoatiara, Niterói, RJ, a census was conducted from January to March 1995. Only canine prevalence of Dirofilaria immitis infection in the district was determined once feline microfilaraemia is rare and transitory and therefore of no epidemiologic importance. Autochthonous cases were demonstrated. Pet population in Itacoatiara was $36 \%$ of human population. It was estimated that in 1995, at Itacoatiara, there were 95 dogs infected by $D$. immitis with microfilaraemia and therefore source of infection. The prevalence of microfilaraemic cases was $31,7 \%$. Among the 91 Itacoatiara born and raised dogs (autochthonous) 37,8\% were microfilaremic.
\end{abstract}

Keywords: Dirofilariasis; Pet population; Heartworm; Dirofilaria immitis.

\section{Introdução}

O conhecimento da população de animais de companhia é imprescindível para o delineamento de programas de controle de doenças, principalmente zoonoses, manejo dessas populações e programas de controle de natalidade. Em geral todos os programas baseiam-se na estimativa da OMS (1989) de que a população de cães e gatos é aproximadamente $10 \%$ da população humana da região. Esta estimativa é generalista e compreende estudos feitos em outros países. No Brasil há poucos estudos que possibilitem o conhecimento do tamanho da população de animais de companhia a partir da população humana. Em Recife, por exemplo, as maiores densidades populacionais humanas e, conseqüentemente, de animais, são encontradas em áreas mais pobres (Lima Junior, 1998).

Itacoatiara é um bairro da Região Oceânica de Niterói que tem como limites o Oceano Atlântico, os maciços costeiros da Serra da Tiririca, divisa com o município de Maricá e o Morro das Andorinhas, separando-o do bairro de Itaipu. $O$ acesso ao bairro se faz por uma única via, que recebe o nome de Mathias Sandri, a partir do posto policial. Tem caráter estritamente residencial, com residências unifamiliares, algumas com a função de veraneio e população residente de 995 habitantes, de renda média alta (CECITEC, 1996).

A dirofilariose canina é conhecida como doença enzoótica na Região Oceânica de Niterói e em 1988 acometia 43,40\% dos cães do lugar (Labarthe et al., 1997). Essa parasitose é causada pelo nematóide Dirofilaria immitis (Leidy, 1856) que habita a luz das artérias pulmonares e do ventrículo direito de cães, embora possa infectar outros hospedeiros tais como seres humanos e felinos (Magalhães, 1887 ; Travassos, 1921 ; Labarthe et al., 1997). Esses parasitas, quando adultos, liberam microfilárias que podem permanecer na circulação do hospedeiro definitivo por até dois anos (Abraham, 1988). Quando os hospedeiros intermediários, que são mosquitos de diferentes espécies (Ludlam et al., 1970), fazem seu repasto sangüíneo, ingerem também as microfilárias. As microfilárias ingeridas passam a parasitar o mosquito e, de-

\footnotetext{
* Bolsistas PIBIC/CNPq/PROPP/UFF.

** Monitora de Anatomia Patológica Veterinária, Departamento de Patologia, MPT/CCM/UFF.

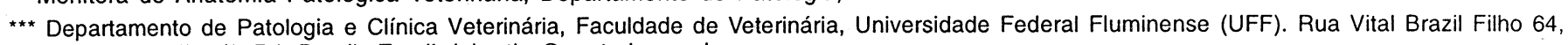
24230-340 Niterói, RJ, Brasil. Email: labarthe @ centroin.com.br.
} 
pendendo das condições climáticas, transformam-se em larvas infectantes (larvas de $3^{\circ}$ estágio) em aproximadamente 20 dias. Quando o mosquito portador das larvas infectantes fizer seu próximo repasto sangüíneo, as larvas migrarão para o tecido subcutâneo do cão e, à medida que migrarem em direção ao coração, mudarão de estágio até chegarem a adultos jovens (100 dias). É no habitat definitivo (coração e artérias pulmonares) que chegam à maturidade sexual e iniciam sua reprodução (Abraham, 1988).

\section{Material e métodos}

Para conhecer o número de cães e gatos de Itacoatiara, Região Oceânica do Município de Niterói, Rio de Janeiro, realizou-se um censo de janeiro a março de 1995. Durante os três meses, as 466 casas do bairro (a partir da Rua das Hortências e Rua dos Gerânios até a Av. Beira Mar) foram visitadas (Figura 1). Nas residências onde houve colaboração por parte do morador, os dadós referentes a cães e gatos de estimação foram registrados em fichas próprias com informações de nome, espécie, idade, local de nascimento, tempo de moradia em Itacoatiara e local de viagens eventuais.

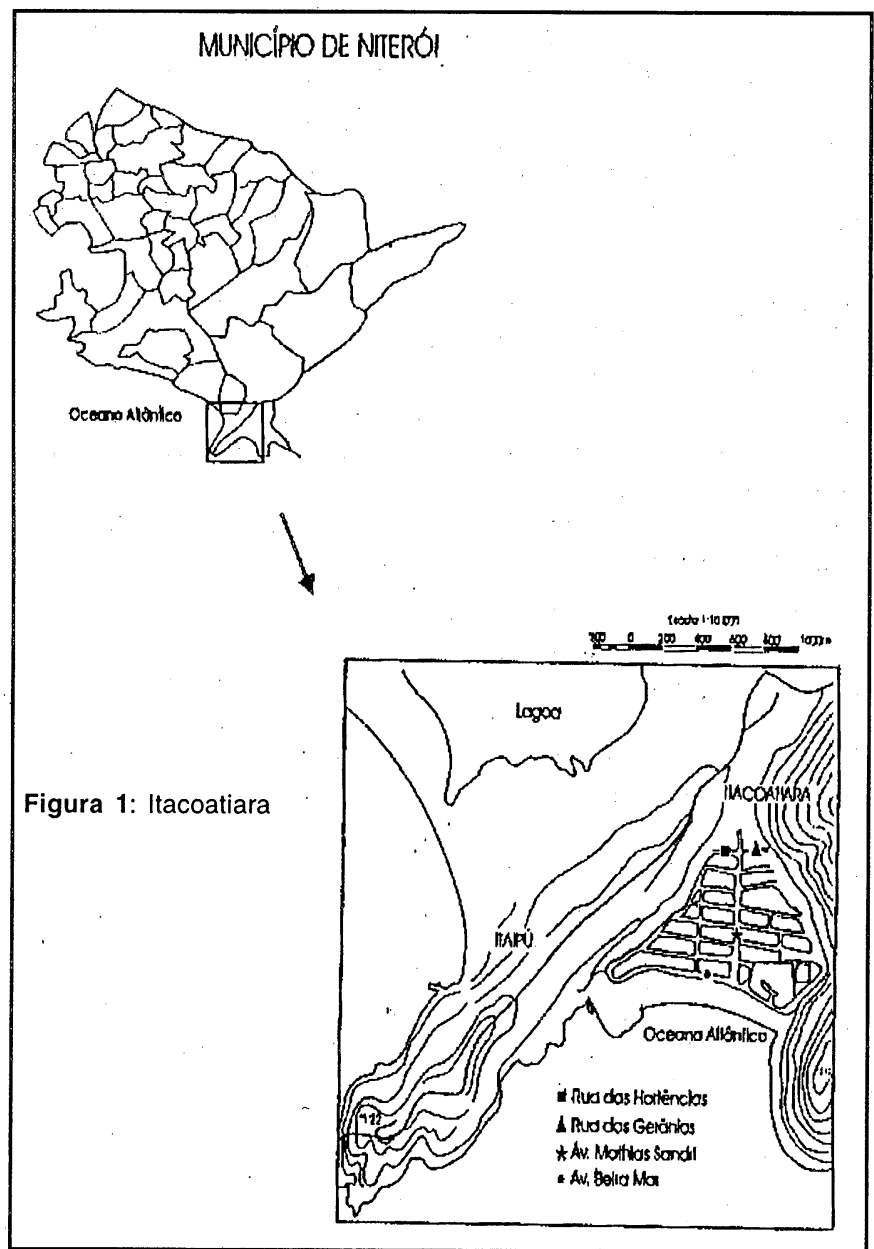

Sempre que os proprietários consentiam, colhia-se sangue dos cães com mais de 1 ano de idade, para pesquisa de microfilárias na circulação. As colheitas foram feitas tanto a domicílio quanto durante a campanha municipal de vacinação contra raiva.

Foram obtidas 123 amostras de sangue, por venopunção cefálica, que foram acondicionadas em tubos contendo EDTA e mantidas a $4^{\circ} \mathrm{C}$ até a realização do exame, na Faculdade de Veterinária da Universidade Federal Fluminense. O diagnóstico foi feito através da identificação de microfilárias de Dirofilaria immitis usando-se as técnicas de Knott (1939), modificada por Newton e Wright (1956) e NAN (Almosny et al., 1991).

Foram considerados casos autóctones, cães portadores de microfilárias de $D$. immitis que nasceram, foram criados em Itacoatiara e que nunca saíram de lá (cães nativos).

O número de cães e gatos das casas sem resposta foi estimado multiplicando-se o número de casas sem resposta pela média de cães e de gatos por casa com resposta. A população de cães e gatos de Itacoatiara foi então calculada somando-se o número de cães e gatos encontrados nas casas com resposta ao número estimado de cães e gatos nas casas sem resposta.

\section{Resultados}

Houve resposta em 416 das 466 casas visitadas. Dessas, em 150 havia 268 cães $(0,64$ cães/casa com resposta e 1,79 cães/casa com cães) e em 36 verificou-se a presença de 53 gatos (0,13 gatos/casa com resposta e 1,47 gatos/casa com gatos). De acordo com esses valores, a população estimada de animais de companhia em Itacoatiara foi de 300 cães e 59 gatos, o que corresponde a $36 \%$ da população humana.

A maioria da população canina de Itacoatiara situou-se na faixa etária entre 2 e 4 anos (Tab. I), assim como aquela que nasceu, cresceu e jamais saiu do bairro (cães nativos) teve maior número de animais neste intervalo.

Foram encontradas larvas de Dirofilaria immitis em 39 das 123 amostras (31,7\%) de sangue analisadas; das 123, 42 amostras (34\%) pertenciam a cães entre 2 e 4 anos de idade. Observou-se que $36 \%$ dos animais nesta faixa etária tinham microfilárias de $D$. immitis. Observou-se também que dentre os cães com menos de 2 anos de idade, somente 1 era portador de microfilárias e que se tratava de caso autóctone (Tabela 1).

Dos 39 casos de dirofilariose, 14 eram confirmadamente autóctones (36\%). Baseando-se no total estimado de cães (300) e na prevalência de dirofilariose encontrada $(31,7 \%)$, pôde-se considerar que no bairro de Itacoatiara, em 1995, provavelmente havia 95 cães infectados por $D$. immitis e portadores de microfilaremia, servindo, portanto, como reservatório.

Trinta e um dos 91 cães nativos foram examinados e 14 deles $(45,2 \%)$ eram portadores de microfilaremia.

A distribuição dos casos positivos entre os grupos etários da população nativa não foi significativa $(p>0,05)$, não havendo relação entre a taxa de infecção e a idade dos cães. 
Tabela 1: População canina por faixa etária e freqüência da infecção por Dirofilaria immitis em cães, em Itacoatiara, município de Niterói, RJ, em 1995

\begin{tabular}{|c|c|c|c|c|c|c|c|c|c|}
\hline \multirow{3}{*}{$\begin{array}{c}\text { Faixa etária } \\
\text { (anos) }\end{array}$} & \multicolumn{3}{|c|}{ Cães introduzidos } & \multicolumn{3}{|c|}{ Cães nativos * } & \multicolumn{3}{|c|}{ Total } \\
\hline & \multirow[t]{2}{*}{ Residentes } & \multicolumn{2}{|c|}{ Examinados } & \multirow[t]{2}{*}{ Residentes } & \multicolumn{2}{|c|}{ Examinados } & \multirow[t]{2}{*}{ Residentes } & \multicolumn{2}{|c|}{ Examinados } \\
\hline & & $\begin{array}{c}\mathrm{MF}+1 \\
\text { total }\end{array}$ & $\%$ & & $\mathrm{MF}+/$ total & $\%$ & & $\begin{array}{c}M F+l \\
\text { total }\end{array}$ & $\%$ \\
\hline$<1$ & 15 & - & - & 15 & - & - & 30 & - & - \\
\hline $1-2$ & 19 & $0 / 9$ & 0 & 11 & $1 / 8$ & 13 & 30 & $1 / 17$ & 6 \\
\hline $2-4$ & 48 & $11 / 32$ & 34 & 23 & $4 / 10$ & 40 & 71 & $15 / 42$ & 36 \\
\hline $4-6$ & 21 & $2 / 13$ & 15 & 13 & $3 / 8$ & 37 & 34 & $5 / 21$ & 24 \\
\hline $6 \cdot 8$ & 36 & $6 / 21$ & 29 & 10 & $1 / 4$ & 25 & 46 & $7 / 25$ & 28 \\
\hline $8-10$ & 20 & $2 / 2$ & 100 & 6 & $2 / 4$ & 50 & 26 & $4 / 6$ & 67 \\
\hline$\geq 10$ & 18 & $4 / 9$ & 44 & 13 & $3 / 3$ & 100 & 31 & $7 / 12$ & 58 \\
\hline TOTAL & 177 & $25 / 86$ & 29 & 91 & $14 / 37$ & 37,8 & 268 & $39 / 123$ & 32 \\
\hline
\end{tabular}

* Cães nativos: cães que nasceram e foram criados em Itacoatiara e nunca saíram de lá.

\section{Discussão e conclusões}

A população de animais de estimação no bairro de Itacoatiara correspondeu a $36,1 \%$ da população humana, o que significa aproximadamente 3,6 vezes a proporção geralmente usada (OMS, 1989). Ao considerar-se que Itacoatiara é um bairro onde predominam famílias de classe média alta e residências unifamiliares, sendo algumas residências de ocupação esporádica (veraneio) e que no Brasil áreas mais pobres tendem a apresentar densidade canina mais elevada (Lima Junior, 1998), pode-se sugerir que a generalização proposta (10\% da população humana) não se aplique.

Itacoatiara é um bairro cujas condições ambientais e paisagem propiciam a transmissão do parasita $D$. immitis. Dentre os fatores que facilitam a transmissão podem ser citados: a abundância em abrigos e criadouros para as diferentes espécies de mosquitos transmissores; a umidade relativa alta; temperatura média variando de 15,6 a $32,3^{\circ} \mathrm{C}$; grande quantidade de cães portadores de microfilaremia (95) e, portanto, fonte de infecção para os mosquitos e grande quantidade de cães susceptíveis à infecção (205). Como mosquitos se alimentam indiscriminadamente em cães, gatos e seres humanos (Lok, 1988), é provável que haja casos de infecções felinas e humanas no bairro. Gatos e seres humanos residentes em áreas enzoóticas de dirofilariose canina estão sujeitos a infecção (Guerrero et al., 1992 ; Campos et al., 1997), principalmente em áreas como Itacoatiara, onde alguns dos mosquitos transmissores do parasita são ecléticos e buscam seres humanos e também gatos, porém com menor freqüência, para fazerem seus repastos sangüineos (Labarthe et al., 1998a,b).

Há vários fatores que podem aumentar o risco de infecção por $D$. immitis para cães. Dentre eles, estão a permanência do lado de fora das residências e o tempo de exposição (Walters, Lavoipierre, 1984). O fato de entre 2 e 4 anos encontrar-se a maior freqüência de cães parasitados sugere que, em Itacoatiara, em poucos anos um cão susceptível estará infectado. Talvez por isso, dentre os cães nativos observa-se uma população jovem, onde a maioria dos animais infectados está entre 1 e 6 anos de idade.

O número de casos de infecção canina por $D$. immitis sabidamente autóctones (36\%) confirma a transmissão ativa do nematóide no bairro.

Considerando-se que em Itacoatiara há grande quantidade de cães portadores de microfilaremia servindo como fonte de infecção, que a população de animais de estimação corresponde a $36 \%$ da população humana e que seres humanos e gatos são hospedeiros acidentais do parasita, supôs-se que haja casos de dirofilariose pulmonar humana, bem como de dirofilariose felina e concluiu-se que, em Itacoatiara, há um número maior de animais de companhia em relação à população humana do que o sugerido pela OMS.

\section{Agradecimentos}

Ao Éder Celestino Eliseu pela ajuda nos trabalhos de campo e à Dra. Ligia Maria Cantarino da Costa pela leitura crítica do manuscrito.

\section{Referências bibliográficas}

ABRAHAM, D. Biology of Dirofilaria immitis. In: BOREHAM, P.F., ATWELL R.B. Dirofilariasis. Florida : CRC Press, 1988. p. 29-46.

ALMOSNY, N.R.P., SOARES, A.M.B., LABARTHE, N.V. Concentration method for detection and morfologic differentiation of live Dirofilaria immitis and Dipetalonema reconditum lanae. Abstracts of the XXIV World Veterinary Congress: 59. Rio de Janeiro, Brazil, 1991.

CAMPOS, J.R.M., BARBAS, C.S.V., FILOMENO, L.T.B., FERNANDEZ, A., MINAMOTO, H., BARBAS FILHO, J.V., JATENEM, F.B. Human Pulmonary Dirofilariasis, Analysis of 24 cases from São Paulo, Brazil. Chest., v. 112, p. 729-733, 1997.
CECITEC - Consultoria Especial de Ciência e Tecnologia / Prefeitura de Niterói, 1996. Niterói Bairros. p. 409-417, 499 p.

GUERRERO, J., McCALL, J.W., DZIMIANSKI, M.T., Mc TIER, T.L., HOLMES, R.A., NEWCOMB, K.M. Prevalence of Dirofilaria immitis infection in cats from the Southeastern United States. Proceedings of the Heartworm Symposium '92.p. 91-95, 1992.

KNOTT, J. A method for making microfilarial surveys on dog blood. Trans. Roy. Soc. Trop. Med. Hyg., v. 33, p. 191-186, 1939.

LABARTHE, N., FERREIRA, A.M.R., GUERRERO, J., NEWCOMB, K., PAES-DE-ALMEIDA, E. Survey of Dirofilaria immitis (Leidy, 1856) in random source cats in metropolitan Rio de Janeiro, Brazil, with descriptions of lesions. Vet. Par., v. 71, p. 301-306, 1997. 
LABARTHE, N., SERRÃO, M.L., MELO, Y.F., OLIVEIRA, S.J., LOURENÇO-DE-OLIVEIRA, R. Mosquito frequency and feeding habits in an enzootic canine dirofilariasis area in Niterói, State of Rio de Janeiro, Brazil. Mem. Inst. Oswaldo Cruz., v. 93, p. 145-154, 1998a.

Potential vectors of Dirofilaria immitis (Leidy 1856) in Itacoatiara, Oceanic Region of Niterói Municipality, State of Rio de Janeiro, Brazil. Mem. Inst. Oswaldo Cruz, v. 93, p. 425-432, 1998b.

LEIDY, J. A synopsis of Entozoa and some of their ecto-congeners observed by the author. Proc. Ac. Natl. Sc. Philadelphia., v. 8, p. 42-58, 1856.

LIMA JUNIOR, A.D. Dinâmica populacional de raiva canina e sua relação com a posse responsável dos animais de estimação. CONGRESSO BRASILEIRO DE BEM ESTAR ANIMAL, 2., 1998, São Paulo, p. 63-67.
LOK, J.B. Dirofilariasp.: Taxonomy and distribution. In: BOREHAM, P.F., ATWELL, R.B. Dirofilariasis. Florida: CRC Press, 1988. p. 2-24.

LUDLAM, K.W., JACHOWSKI, L.A., OTTO, G.F. Potential vectors of Dirofilaria immitis. JAVMA., v. 157, p. 1354-1359, 1970.

MAGALHÃES, P.S. Descripção de uma espécie de filárias encontradas no coração humano. Rev. Cursos Prat. Theor. Fac. Med. Rio de Janeiro., v. 3, p. 129-215, 1887.

NEWTON, W.L., WRIGHT, W.H. The occurrence of a dog filariid other than Dirofilaria immitis in the United States. J. Parasitol., v. 42, p. 246-256, 1956.

TRAVASSOS, L. Notas helmintológicas. Brazil Med., v. 2, p. 67, 1921.

WALTERS, L.L., LAVOIPIERRE, M.M.J. Landscape epidemiology of mosquito-borne canine heartworm (Dirofilaria immitis) in Northern California, USA. J. Med. Entomol., v. 21, p. 1-16, 1984. 